\title{
miR-155 is upregulated in patients with active tuberculosis and inhibits apoptosis of monocytes by targeting FOXO3
}

\author{
JIAN HUANG ${ }^{1}$, JUNHUA JIAO ${ }^{2}$, WEIHUA XU ${ }^{2}$, HUAYANG ZHAO ${ }^{3}$, \\ CHUNXIAO ZHANG ${ }^{3}$, YAN SHI ${ }^{1}$ and ZHIJIAN XIAO ${ }^{1}$ \\ ${ }^{1}$ Second Tuberculosis Internal Medicine Department; ${ }^{2}$ First Tuberculosis Internal Medicine Department; \\ ${ }^{3}$ Third Tuberculosis Internal Medicine Department, The First Affiliated Hospital of Xinxiang Medical University, \\ Weihui, Henan 453100, P.R. China
}

Received October 24, 2014; Accepted July 21, 2015

DOI: $10.3892 / \mathrm{mmr} .2015 .4250$

\begin{abstract}
The aim of the present study was to investigate the association between microRNA (miR)-155 and apoptosis of monocytes infected by Mycobacterium tuberculosis, to examine the effect of forkhead box O3 (FOXO3) on miR-155. The present study analysed the apoptosis of $\mathrm{CD}_{14}{ }^{+}$in the peripheral blood of patients with active tuberculosis, disposed the THP-1 human monocytic cell line by BCG and examined the expression of miR-155. Furthermore, the expression of FOXO3 in THP-1 cells was determined, and wild- and mutant-type luciferase reporter plasmids containing FOXO3 3'-untranslated regions (UTRs) were constructed to analyse the expression of luciferase. Finally, an over-expression plasmid was constructed, and THP-1 cells were transfected with control miRNA, miR-155 and the plasmid, which revealed that miR-155 inhibited the apoptosis of THP-1 cells. miR-155 in the THP-1 cells infected by BCG was upregulated and apoptosis also increased. However, the apoptosis declined when the cells were transfected with the control miRNA and miR-155. Folllowing transfection with miR-155, the expression of FOXO3 decreased. Transfection with miR-155 and the FOXO3 3'-UTRs significantly reduced luciferase, and overexpression of FOXO3 reversed the inhibitory role of miR-155. From these results, it was concluded that mycobacteria can improve the level of miR-155, while BCG can induce apoptosis in THP-1 cells. The results suggested FOXO3 is a downstream target gene of miR-155, which combines 3'-UTRs to inhibit the expression of FOXO3.
\end{abstract}

Correspondence to: Professor Zhijian Xiao, Second Tuberculosis Internal Medicine Department, The First Affiliated Hospital of Xinxiang Medical University, 88 Health Road, Weihui, Henan 453100, P.R. China

E-mail: 406741285@qq.com

Key words: tuberculosis, monocyte, microRNA-155, FOXO3

\section{Introduction}

Tuberculosis (TB) is a major infectious disease worldwide, from which millions of people are affected each year and has the second highest mortality rates of infectious diseases (1). The control of TB infection requires innate and adaptive immune responses $(2,3)$. The cellular immunity response of host cells determines whether the infection becomes a latent TB infection (LTBI) or progresses into active TB (ATB). Effective cell-mediated immunity can maintain the TB infection permanently at the LTBI stage, however, if infected individuals cannot control the initial pulmonary infection, or the immune system is weakened, Mycobacterium tuberculosis may cause pulmonary or extrapulmonary tuberculosis (4). The only effective vaccine is the Bacille Calmette-Guerin vaccine (BCG) vaccine), which has limited protective effects and is a major contributor to the incidence of TB increasing over past decades (5). Studies have demonstrated that BCG can induce apoptosis in THP-1 cells (6). There are $\sim 90 \%$ individuals infected with Mycobacterium tuberculosis, who will stay in asymptomatic LTBI stage, with only $10 \%$ individuals exhibiting active tuberculosis, indicating that host genetic factors are important in the regulation of TB infection (7).

MicroRNAs (miRNAs) are evolutionary conserved small RNAs in eukaryotic organisms, which are involved in post-transcriptional regulation (8). Various studies have found that the abnormal expression of miRNAs is associated with numerous human diseases, such as lung cancer, renal cell carcinoma and inflammation (9-12). There is evidence to indicate that certain miRNAs are involved in the regulation of the cellular immune process, therefore, they can be used as potential immune disorder biomarkers (13). For example, miRNA (miR)-146a is expressed largely in human memory $\mathrm{T}$ cells, however, it decreases in naïve $\mathrm{T}$ cells and results in activation of $\mathrm{T}$ cell receptors in the initial T lymphocytes (14). Studies have also demonstrated that miR-144* is predominantly expressed in T cells, and its overexpression in patients with ATB suggests that specific miRNAs may be involved in the biological behavior of T cells $(15,16)$.

miR-155 has been identified as a multifunctional miRNA, which is involved in several biological processes, including tumour growth, infection, inflammation and immunity $(17,18)$. A previous study found that miR-155 is dysregulated in 
human macrophages infected by different Mycobacterium species (19). The expression of miR-155 can enhance the levels of tumour necrosis factor by increasing mRNA stability and half-life (20).

Forkhead box O3 (FOXO3) is one of the forkhead transcription factors, which is involved in regulating cell cycle and innate immune responses, and resisting oxidation and cell apoptosis (21-24). Evidence indicates that BCG-mediated apoptosis of human macrophages is dependent on the activation of FOXO3 transcription factor, and cell apoptosis induced by $\mathrm{BCG}$ is associated with prosurvival-activated threoninekinase dephosphorylation and its target FOXO3, with transfer of FOXO3 to the nucleus of BCG-infected cells leading to increased transcriptional activity of FOXO3 (25). Investigation involving breast cancer cell lines demonstrated that FOXO3 is the direct target of miR-155, and miR-155 is closely associated with the expression of FOXO3 (26).

Another previous study has confirmed that miR-155 induces cell proliferation by inhibiting apoptosis and promoting metastasis, as well as the invasion of glioma cells. However, miR-155 has no effect on cell cycle, but can reduce the expression of FOXO3a by directly targeting its 3'-untranslated regions (3'-UTRs) (27).

\section{Materials and methods}

Human subjects. A total of 20 patients diagnosed with TB were recruited from the Department of Tuberculosis, the First Affiliated Hospital of Xinxiang Medical College (Xinxiang, China). The diagnosis of tuberculosis was based on serum smear acid-fast staining or bacterial cultures, chest X-ray, clinical symptoms and response to anti-TB treatment. In addition, 17 healthy control individuals were randomly selected from a routine physical examination of healthy individuals at the First Affiliated Hospital of Xinxiang Medical College. The inclusion criteria included the following: A normal physical examination result, no fever, cough or other active TB symptoms. The study protocols were approved by the Ethics Committee of the First Affiliated Hospital of Xinxiang Medical College, and informed written consent was obtained from all participants prior to commencement.

Flow cytometric analysis. At 8:00 am, peripheral blood samples $(5 \mathrm{ml})$ were drawn from the antecubital vein of the patients with ATB and the healthy control individuals, and surface antibody was stained within $6 \mathrm{~h}$ with fluorescein isothiocyanate (FITC)-labeled mouse anti-human $\mathrm{CD}_{14}{ }^{+}$monoclonal antibody [HCD14 (cat. no. 301803); BioLegend, Inc., San Diego, CA, USA], incubated for $30 \mathrm{~min}$ on ice, in the dark (dilution, 1:100). The appropriate homeotypic antibody was used to determine the background level of staining. At least 100,000 events were collected and analysed using Beckman CXP (version 2.0) software on a FC-500 Flow Cytometer (Beckman Coulter, Brea, CA, USA). The content of $\mathrm{CD}_{14}{ }^{+}$was analysed using flow cytometric analysis. In addition, samples from the ATB patients and control individuals were obtained to determine the levels of apoptosis. The experiments were performed in triplicate.

Reverse transcription-quantitative polymerase chain reaction $(R T-q P C R)$. Density gradient centrifugation was performed, at $1,500 \mathrm{x} \mathrm{g}$ at $4^{\circ} \mathrm{C}$ for $5 \mathrm{~min}$, using Ficoll-Paque (GE Healthcare Biosciences, Pittsburgh, PA, USA) to purify the peripheral blood mononuclear cells (PBMCs). Subsequently, RT-qPCR was performed to analyse the expression of miR-155 in the PBMCs of the ATB patents and control individuals. Total RNA was extracted using TRIzol reagent (Invitrogen Life Technologies, Carlsbad, CA, USA), according to the manufacturer's instruction. Complementary deoxyribonucleic acid (cDNA) was synthesised from the total RNA using a PrimeScript II First Strand cDNA Synthesis kit (Takara Biotechnology, Co., Ltd., Dalian, China), according to standard protocol. qPCR was then performed on the cDNA using SYBR Green dye (Takara Biotechnology, Co., Ltd.) with specific primers as follows: Forward, TGAACCTCTGCTGCCCATAC and reverse, GCCACCTTCAAGAAGTAGCCTAT (MGH DNA Core Facility, Cambridge, MA, USA). Briefly, $25 \mathrm{ml}$ SYBR Green Mix (2X), $0.5 \mathrm{ml}$ cDNA, $2 \mathrm{ml}$ primer pair mix (5 pmol/ml each primer) and $22.5 \mathrm{ml} \mathrm{H}_{2} \mathrm{O}$ were used. The thermocycling conditions were as follows: $50^{\circ} \mathrm{C}$ for $2 \mathrm{~min}$ (1 cycle), $95^{\circ} \mathrm{C}$ for $10 \mathrm{~min}$ ( 1 cycle), followed by 40 cycles of $95^{\circ} \mathrm{C}$ for $15 \mathrm{sec}, 60^{\circ} \mathrm{C}$ for $30 \mathrm{sec}$ and $72^{\circ} \mathrm{C}$ for $30 \mathrm{sec}$ and finally, $72^{\circ} \mathrm{C}$ for $10 \mathrm{~min}$ ( 1 cycle). U6 served as an internal control. The median in each triplicate was used to calculate the relative content using the comparative $\Delta \mathrm{Ct}$ method (value of $2^{-\Delta \mathrm{Ct}}$ ) (28). The fold changes in expression were calculated using $2^{-\Delta \Delta \mathrm{Ct}}$ methods. The THP-1 human monocytic cell line was obtained from Shanghai Institute of Cell Biology (Shanghai, China). The cells were cultured in RPMI-1640 medium supplemented with $10 \%$ fetal bovine serum (Shiyi Biotechnology, Inc., Shanghai, China). The cells were cultured in a humidified incubator at $37^{\circ} \mathrm{C}$ with $5 \%$ carbon dioxide. The THP-1 cells (density, 10,000/ml) were divided into a treatment group, which was exposed to BCG (multiplicity of infection=5) for $4 \mathrm{~h}$, and a control group. The cells were cultured for $24 \mathrm{~h}$ at $37^{\circ} \mathrm{C}$, and the expression of miR-155 was analysed using RT-qPCR according to the above-mentioned method. The cells in the treatment group were transfected with control miRNA and miR-155 mimics [either small interfering (si)RNA oligonucleotides or scrambled siRNA controls with Lipofectamine 2000 (Invitrogen Life technologies) according to the standard protocol], and apoptosis was assessed using flow cytometry (FITC Annexin V Apoptosis Detection kit; BD Biosciences, San Jose, CA, USA). Briefly, camptothecin was added (at a final concentration of 4-6 $\mu \mathrm{M}$ ) to $1 \times 10^{6}$ cells. The cells were then incubated for 4-6 h at $37^{\circ} \mathrm{C}$, which was followed by the FITC Annexin V and propidium iodide staining protocol (Beyotime Institute of Biotechnology, Haimen, China) to measure apoptosis. The experiments were performed in triplicate.

Western blot analysis and luciferase assay. The THP-1 cells were cultured for $24 \mathrm{~h}$ at $37^{\circ} \mathrm{C}$ and then transfected with control miRNA and miR-155 mimics using Lipofectamine 2000, according to the manufacturer's instructions. Following incubation of the cells for $48 \mathrm{~h}$, the expression level of FOXO3 was analysed using western blot analysis, according to the standard protocols. Briefly, cells were lysed in precipitation assay buffer (Sigma-Aldrich, St. Louis, MO, USA) and sonicated (duration, $15 \mathrm{sec}$; amplitude, 30\%) using a Sonics Vibra-Cell 
Table I. Demographic and clinical characteristics in patients with ATB and healthy control individuals.

\begin{tabular}{lcc}
\hline Characteristic & TB patients $(\mathrm{n}=20)$ & Healthy controls $(\mathrm{n}=17)$ \\
\hline Gender (male/female) & $12 / 11$ & $10 / 7$ \\
Age (years; mean \pm SD) & $54.32 \pm 18.17$ & $49.17 \pm 16.36$ \\
Pulmonary TB (n) & $20 / 20$ & - \\
Culture-positive TB (n) & $20 / 20$ & - \\
Smear-positive TB (n) & $9 / 20$ & - \\
Tuberculin skin test (n) & & - \\
$>10$ cm & 5 & - \\
$>5$ cm & 6 & - \\
Elevated ESR (n) & $12 / 20$ & - \\
Fever (n) & $7 / 20$ & - \\
MDR/XDR TB (n) & $4 / 20$ & $17 / 17$ \\
BCG vaccinated (n) & $20 / 20$ & \\
\hline
\end{tabular}

ATB, active tuberculosis; TB, tuberculosis; ESR, erythrocyte sedimentation rate; MDR, multi-drug resistance; XDR, extensively drug-resistant; $\mathrm{BCG}$, Bacille Calmette-Guerin vaccine; SD, standard deviation.

(Sonics Materials, Inc. Newtown, CT, USA) and centrifuged at $16,100 \mathrm{x} \mathrm{g}$ for $15 \mathrm{~min}$ at $4^{\circ} \mathrm{C}$. Protein concentrations in lysates were measured via BCA assay (Pierce Biotechnology, Inc., Rockford, IL, USA). Equal quantities of protein were separated in $8 \%$ SDS-PAGE (Shanghai Bogu Biological Technology Co., Ltd., Shanghai, China) and transferred onto polyvinylidene fluoride membranes (Bio-Rad Laboratories, Inc., Hercules, CA, USA). Membranes were blocked overnight at $4^{\circ} \mathrm{C}$, incubated with the primary antibody (anti-FOXO3A; cat. no. Ab23683; Abcam, Cambridge, UK; dilution 1:1,000) and the secondary antibody (HRP-labeled goat anti-rabbit IgG; cat. no. Ab6720; Abcam; dilution 1:2,500) in 0.1\% Tween-20 (Nanjing Sen Beijia Biological Technology Co., Ltd., Nanjing, China) with $2 \%$ skimmed milk in phosphate-buffered saline or $5 \%$ bovine serum albumin (Shanghai Bogu Biological Technology Co., Ltd.) in Tris-buffered saline (Shanghai Bogu Biological Technology Co., Ltd.). The chemiluminescent signal was detected using a SuperSignal West Femto Substrate (Thermo Fisher Scientific, Inc., Waltham, MA, USA) and ImageQuant 400 imaging system (GE Healthcare Life Sciences). Data were normalised to GAPDH. A wild-type and mutant luciferase reporter plasmid containing FOXO3 3' end non-coding region were constructed and cotransfected with the miR-155 mimics and FOXO3, and the miR-155 inhibitor and FOXO3, respectively, to observe the expression of luciferase. Briefly, gene expression was silenced using FOXO3 siRNA (GE Dharmacon, Chicago, IL, USA) and transfection with siRNAs or plasmid DNAs was performed using Lipofectamine 2000 according to the manufacturer's instructions. The transfected cells were then treated with palmitic acid (Sigma-Aldrich) at the designated concentrations for the indicated durations. The experiments were performed in triplicate.

Over expression of $\mathrm{FOXO3.} \mathrm{An} \mathrm{overexpression} \mathrm{plasmid} \mathrm{was}$ constructed from the 3 ' end non-coding region of FOXO3, The THP-1 cells were contransfected with the control miRNA, miR-155 mimic and FOXO3 overexpression plasmid following treatment with BCG. The expression level of FOXO3 was
A

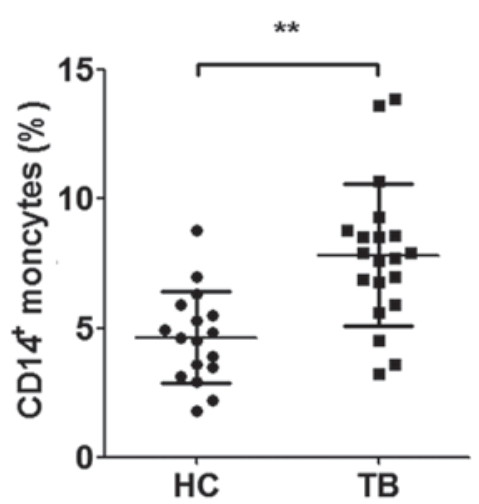

B

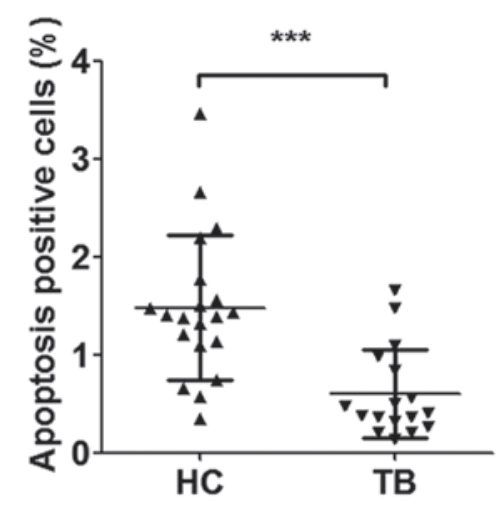

Figure 1. (A) Percentage of peripheral blood CD14 ${ }^{+}$monocytes of patients with ATB was significantly increased, compared with the healthy controls $\left({ }^{* *} \mathrm{P}<0.01\right)$. (B) Percentage of apoptosis-positive cells in patients with ATB was significantly reduced, compared with healthy controls $\left({ }^{* * *} \mathrm{P}<0.001\right)$. HC, healthy control; TB, tuberculosis.

analysed using western blot analysis, and changes in apoptosis in the THP-1 cells were determined using flow cytometric analysis (according to the above-mentioned procedures). The experiments were performed in triplicate. 
A

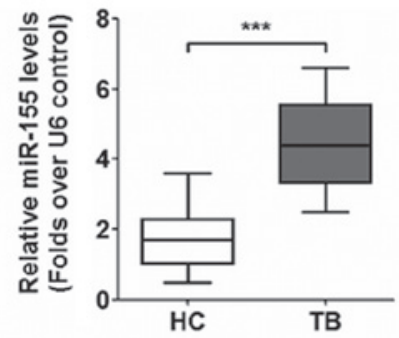

B

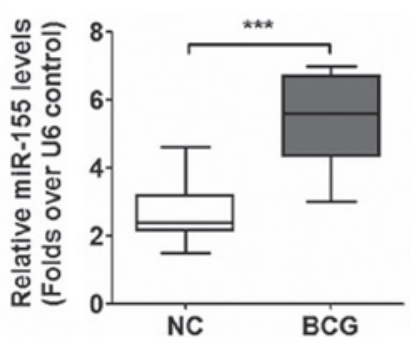

C

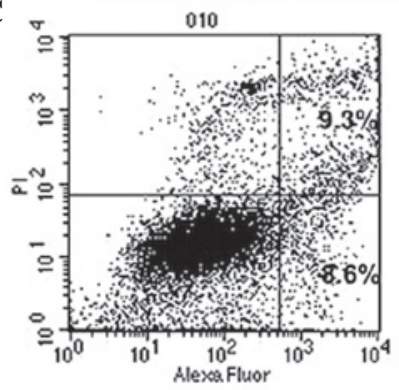

miR-con mimics

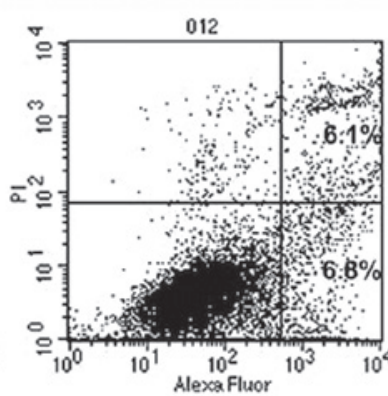

miR-155 mimics
D

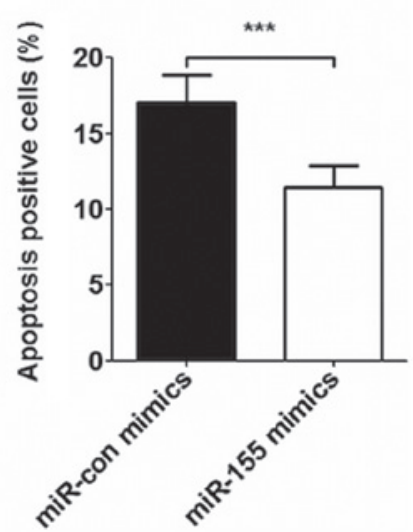

Figure 2. (A) Reverse transcription-quantitative polymerase chain reaction analysis revealed that the relative expression level of miR-155 in patients with ATB was significantly higher than in healthy controls $\left({ }^{* * * *} \mathrm{P}<0.001\right)$. (B) Following infection with BCG, the relative expression level of miR-155 in THP-1 cells was elevated significantly $\left.{ }^{* * *} \mathrm{P}<0.001\right)$. (C) Apoptosis assay revealed that, following transfection with miR-155 mimics, the apoptosis of the THP-1 cells decreased $1.8 \%$, compared to transfection with miR-con mimics. (D) Apoptosis of THP-1 cells transfected with miR-155 mimics reduced significantly ( $\left.{ }^{* * *} \mathrm{P}<0.001\right)$. The data are presented as the mean \pm standard deviation. HC, healthy control; NC, normal control; TB, tuberculosis; miR, microRNA; con, control; PI, propidium iodide.

A

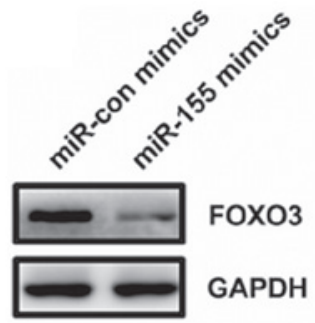

B

FOXO3 3'UTR

hsa-miR-155

5'......UUUCUUUGCAUAAAAAGCAUUAG.......3'

3'.......UGGGGAUAGUGCUAAUUCGUA'I İU......

Mut FOXO3 3'UTR

hsa-miR-155

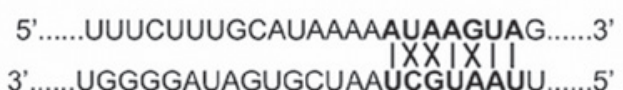

C

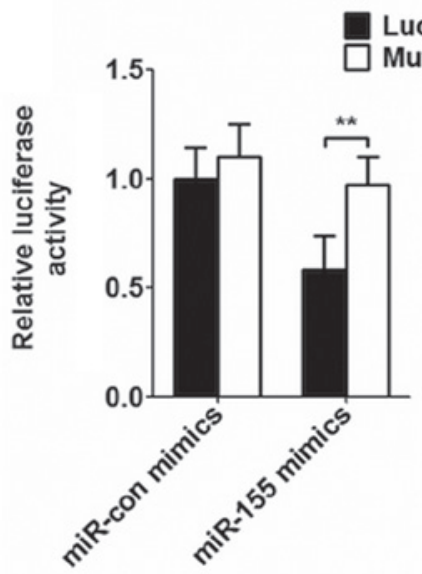
Luc FOXO3 3'-UTR
Mut FOXO3 3'-UTR
D

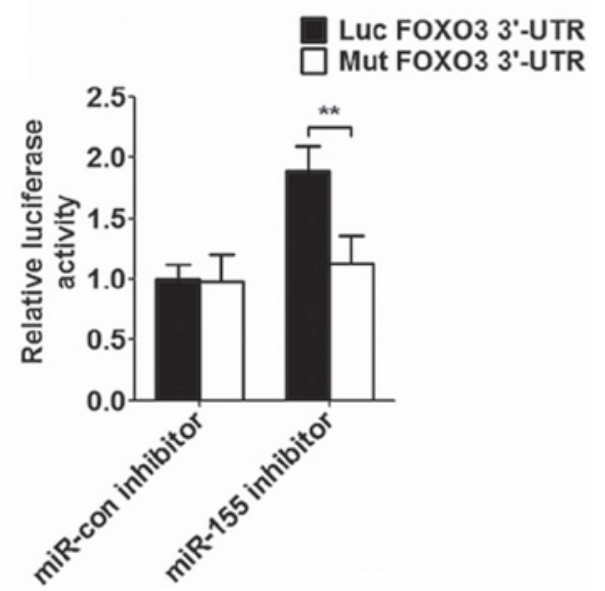

Figure 3. (A) Western blot revealed that the expression level of FOXO3 in cells transfected with miR-155 mimics decreased. (B) Alignment between the predicted miR-155 target site of the FOXO3 3'-UTR region and miR-155. (C) Wild-type luciferase reporter plasmid containing FOXO3 3'-end non-coding region transfected with miR-155 mimics and FOXO3 reduced the expression of luciferase $\left({ }^{* *} \mathrm{P}<0.01\right)$. No significant difference was observed following transfection with miR-con mimics ( $\mathrm{P}>0.05)$. (D) Luciferase activity was significantly increased following transfection with miR-155 inhibitor and FOXO3 (** $<<0.01)$, while no significant difference was observed following transfection with the miR-con mimics $(\mathrm{P}>0.05)$. The data are presented as the mean \pm standard deviation. FOXO3, forkhead box O3; HC, healthy control; TB, tuberculosis; miR, microRNA; con, control; Mut, mutant; Luc, luciferase. 
A

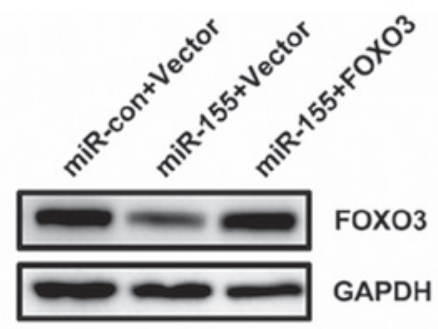

B

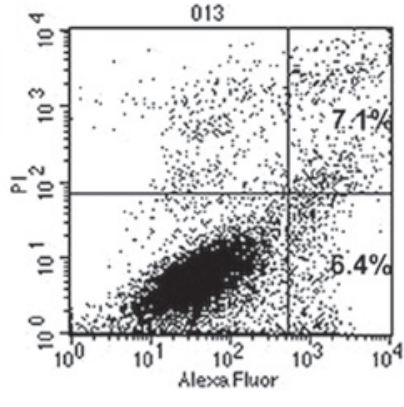

miR-155+Vector

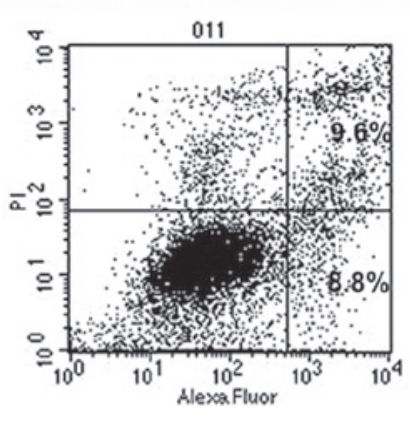

miR-155+FOXO3
C

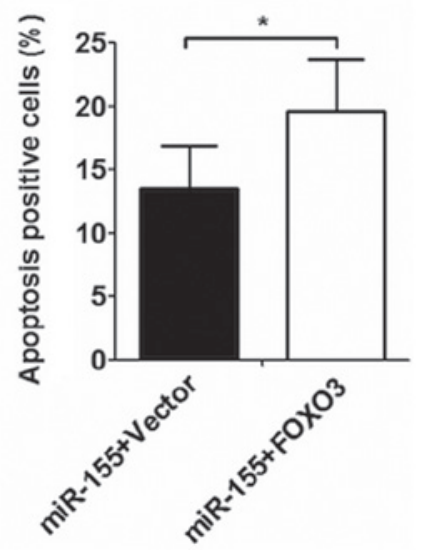

Figure 4. (A) Western blot revealed that the expression of FOXO3 decreased following transfection with miR-155 mimics, while there was no change following transfection with miR-con and miR-155 in combination with FOXO3. (B) Assay of apoptosis indicated that the apoptosis increased when the cells were transfected with miR-155 in combination with FOXO3, compared with those transfected with miR-155 mimics. (C) Quantitative results revealing the percentage of apoptosis increased when the cells were transfected with miR-155 in combination with FOXO3, compared with those transfected with miR-155 mimics $($ * $\mathrm{P}<0.05)$. The data are presented as the mean \pm standard deviation. FOXO3, forkhead box O3; miR, microRNA; con, control.

Statistical analysis. All the data are presented as the mean \pm standard deviation. The differences between groups were assessed using a two-tailed non-paired Student's $t$-test or using one-way analysis of variance. $\mathrm{P}<0.05$ was considered to indicate a statistically significant difference. All statistical analyses were performed on a personal computer with the SPSS statistical package version 16.0 (SPSS, Inc., Chicago, IL, USA) for Windows.

\section{Results}

Apoptosis is reduced in the PBMCs of patients with ATB. The data regarding the clinical and demographic characteristics are presented in Table I. The number of PBMCs of the ATB patients was significantly increased, compared with the healthy controls (Fig. 1A). Analysis of the apoptosis of CD14 ${ }^{+}$ monocytes was performed using a flow cytometric assay, which demonstrated a significant reduction in the level of apoptosis in the PBMCs of patients with ATB, compared with the healthy controls (Fig. 1B).

Expression of miR-155. In the PBMCs of patients with ATB, the expression of miR-155 is increased significantly, compared with that observed in the PBMCs of the healthy control individuals (Fig. 2A). Following treatment of the THP-1 mononuclear cell line with BCG (MOI=5) for $4 \mathrm{~h}$ in solution and incubation for $24 \mathrm{~h}$, quantitative analysis revealed that the level of miR-155 was elevated following being infected (Fig. 2B), compared with the control cells without BCG. The present study also transfected the THP-1 cells with control miRNA and miR-155 mimics, and subsequent flow cytometry was performed to observe the levels of apoptosis. The results of this analysis revealed that the apoptosis of the cells reduced following transfection with the miR-155 mimics, compared with those transfected with the control (Fig. 2C and D).

miR-155 decreases the expression of FOXO3. Following transfection of the cells with miR-155, the expression of FOXO3 decreased, compared with the miRNA control cells (Fig. 3A). In addition, the results of the luciferase assay revealed that cotransfection with the wild-type luciferase reporter plasmid, containing the FOXO3 3'-end non-coding region, and miR-155 and FOXO3 decreased the activity of luciferase (Fig. 3B and C). By contrast, the activity of luciferase was markedly increased following transfection with miR-155 inhibitor and FOXO3 (Fig. 3D).

FOXO3 reverses the effect of miR-155 on THP-1 apoptosis. The expression of FOXO3 in the THP-1 cells cotransfected with control miRNA, miR-155 mimics and the FOXO3 overexpression plasmid following treating with BCG were also analysed in the present study. The results revealed that the expression of FOXO3 decreased following transfection with miR-155 mimics (Fig. 4A), however, there was no change in expression following transfection with the miRNA control or miR-155 in combination with FOXO3. The assay to determine apoptosis demonstrated that the level of apoptosis increased when the cells were transfected with miR-155 in combination with $\mathrm{FOXO} 3$, which indicated that the overexpression of FOXO3 significantly reversed the effect of miR-155 on THP-1 cell apoptosis (Fig. 4B and C). 


\section{Discussion}

Peripheral blood in the blood of patients with ATB contains various differences to that of healthy individuals in terms of miRNAs, and a previous study revealed that monocytes grow in quantity in patients with ATB (29), with the expression of miR-155 demonstrating the same trend (30). In the present study, the number of monocytes in patients with ATB were analysed and its association with the expression level of miR-155 was investigated. First, the present study confirmed that the number of CD14 ${ }^{+}$monocytes in the peripheral blood of patients with ATB increased significantly, whereas the levels of apoptosis markedly decreased. In addition, the expression of miR-155 in the PMBCs of the patients with ATB, as well as the expression of miR-155 in THP-1 cells infected by BCG increased significantly, which demonstrated that mycobacterial infection may elevate the level of miR-155. A previous study verified that BCG can induce the apoptosis of THP-1 cells (6). In the present study, the transfection of THP-1 cells with miRNA and miR-155 mimics was performed following treatment with BCG. Subsequent analyses led to the conclusion that miR-155 mimics inhibited the apoptosis of THP-1 cells. Through the assessment of molecular information, the present study established that FOXO3 is a downstream target gene of miR-155, and a previous report demonstrated that FOXO3 is involved in apoptosis caused by infection with mycobacterium (25). In the present study, the THP-1 cells were transfected with miRNA control and miR-155 mimics at the same time. It was demonstrated that, following the transfection with miR-155, the expression of FOXO3 decreased. In addition, cotransfection of a wild-type luciferase reporter plasmid containing the FOXO3 3 ' end non-coding region with miR-155 and FOXO3 was observed to lessen the expression of luciferase. By contrast, the expression of luciferase markedly increased when the cells were transfected with miR-155 inhibitor and FOXO3, which indicated that miR-155 may inhibit the expression of FOXO3 by combining with the FOXO3 3'-end non-coding region. Furthermore, the present study constructed an overexpression plasmid from the 3 '-end non-coding region of FOXO3, and the THP-1 cells were contransfected with control miRNA, miR-155 mimics and the FOXO3 overexpression plasmid following treatment with BCG. The results revealed that overexpression of FOXO3 significantly reversed the effect of miR-155 on THP-1 cell apoptosis, which demonstrated that the inhibition of miR-155 on monocyte apoptosis was associated with the regulation of FOXO3 target genes.

In conclusion, the results of the present study confirmed that mononuclear cells in the peripheral blood of patients with ATB increase as a result of apoptosis being suppressed which is induced by the expression of miR-155. The results also demonstrated that inhibitory effect of miR-155 on monocyte apoptosis occurs through the regulation of FOXO3 target genes.

\section{References}

1. WHO: Global tuberculosis report. World Health Organization, France: 1-89, 2012.

2. Cooper AM: Cell-mediated immune responses in tuberculosis. Annu Rev Immunol 27: 393-422, 2009.
3. Natarajan K, Kundu M, Sharma P and Basu J: Innate immune responses to $M$. tuberculosis infection. Tuberculosis (Edinb) 91: 427-431, 2011.

4. Smith I: Mycobacterium tuberculosis pathogenesis and molecular determinants of virulence. Clin Microbiol Rev 16: 463-496, 2003.

5. Russell DG, Barry CE III and Flynn JL: Tuberculosis: What we don't know can and does, hurt us. Science 328: 852-856, 2010.

6. Riendeau CJ and Kornfeld H: THP-1 cell apoptosis in response to Mycobacterial infection. Infect Immun 71: 254-259, 2003.

7. Khalilullah SA, Harapan H, Hasan N, Winardi W, Ichsan I and Mulyadi M: Host genome polymorphisms and tuberculosis infection: What we have to say? Egyptian Journal of Chest Diseases and Tuberculosis 63: 173-85, 2014.

8. Akita T, Takuno S and Innan H: Modeling evolutionary growth of a microRNA-mediated regulation system. J Theor Biol 311: 54-65, 2012.

9. Kanwar JR, Mahidhara G and Kanwar RK: MicroRNA in human cancer and chronic inflammatory diseases. Front Biosci (Schol Ed) 2: 1113-1126, 2010.

10. Chen XM: MicroRNA signatures in liver diseases. World J Gastroenterol 15: 1665-1672, 2009.

11. Christensen $M$ and Schratt GM: MicroRNA involvement in developmental and functional aspects of the nervous system and in neurological diseases. Neurosci Lett 466: 55-62, 2009.

12. Pauley KM, Cha S and Chan EK: MicroRNA in autoimmunity and autoimmune diseases. J Autoimmun 32: 189-194, 2009.

13. Rodriguez A, Vigorito E, Clare S, Warren MV, Couttet P, Soond DR, van Dongen S, Grocock RJ, Das PP, Miska EA, et al: Requirement of bic/microRNA-155 for normal immune function. Science 316: 608-611, 2007.

14. Curtale G, Citarella F, Carissimi C, Goldoni M, Carucci N, Fulci V, Franceschini D, Meloni F, Barnaba V and Macino G: An emerging player in the adaptive immune response: MicroRNA-146a is a modulator of IL-2 expression and activation-induced cell death in T lymphocytes. Blood 115: 265-273, 2010.

15. Liu Y, Wang X, Jiang J, Cao Z, Yang B and Cheng X: Modulation of $\mathrm{T}$ cell cytokine production by miR-144* with elevated expression in patients with pulmonary tuberculosis. Mol Immunol 48: 1084-1090, 2011.

16. Marín ND, París SC, Rojas M and García LF: Reduced frequency of memory T cells and increased Th17 responses in patients with active tuberculosis. Clin Vaccine Immunol 19: 1667-1676, 2012.

17. Tsitsiou E and Lindsay MA: MicroRNAs and the immune response. Curr Opin Pharmacol 9: 514-520, 2009.

18. Hu R, Kagele DA, Huffaker TB, Runtsch MC, Alexander M, Liu J, Bake E, Su W, Williams MA, Rao DS, et al: miR-155 promotes $\mathrm{T}$ follicular helper cell accumulation during chronic, low-grade inflammation. Immunity 41: 605-619, 2014.

19. Rajaram MV, Ni B, Morris JD, Brooks MN, Carlson TK, Bakthavachalu B, Schoenberg DR, Torrelles JB and Schlesinger LS: Mycobacterium tuberculosis lipomannan blocks TNF biosynthesis by regulating macrophage MAPK-activated protein kinase 2 (MK2) and microRNA miR-125b. Proc Natl Acad Sci USA 108: 17408-17413, 2011.

20. Bala S, Marcos M, Kodys K, Csak T, Catalano D, Mandrekar P and Szabo G: Up-regulation of microRNA-155 in macrophages contributes to increased tumor necrosis factor $\{$ alpha\} (TNF\{alpha\}) production via increased mRNA half-life in alcoholic liver disease. J Biol Chem 286: 1436-1444, 2011.

21. Brunet A, Bonni A, Zigmond MJ, Lin MZ, Juo P, Hu LS, Anderson MJ, Arden KC, Blenis J and Greenberg ME: Akt promotes cell survival by phosphorylating and inhibiting a Forkhead transcription factor. Cell 96: 857-868, 1999.

22. Arden KC: FOXO animal models reveal a variety of diverse roles for FOXO transcription factors. Oncogene 27: 2345-2350, 2008.

23. Peng SL: Foxo in the immune system. Oncogene 27: 2337-2344, 2008.

24. Ticchioni M, Essafi M, Jeandel PY, Davi F, Cassuto JP, Deckert M and Bernard A: Homeostatic chemokines increase survival of B-chronic lymphocytic leukemia cells through inactivation of transcription factor FOXO3a. Oncogene 26: 7081-7091, 2007.

25. Haoues M, Refai A, Mallavialle A, Barbouche MR, Laabidi N, Deckert M and Essafi M: Forkhead box O3 (FOXO3) transcription factor mediates apoptosis in BCG-infected macrophages. Cell Microbiol 16: 1378-1390, 2014.

26. Harada Y, Harada Y, Elly C, Ying G, Paik JH, DePinho RA and Liu YC: Transcription factors Foxo3a and Foxol couple the E3 ligase Cbl-b to the induction of Foxp3 expression in induced regulatory T cells. J Exp Med 207: 1381-1391, 2010. 
27. Ling N, Gu J, Lei Z, Li M, Zhao J, Zhang HT and Li X: MicroRNA-155 regulates cell proliferation and invasion by targeting FOXO3a in glioma. Oncol Rep 30: 2111-2118, 2013.

28. Zhang L, Liu S, Zhang L, You H, Huang R, Sun L, He P, Chen S, Zhang $\mathrm{H}$ and Xie P: Real-time qPCR identifies suitable reference genes for Borna disease virus-infected rat cortical neurons. Int J Mol Sci 15: 21825-21839, 2014.
29. Liu Y, Jiang J, Wang X,Zhai F and Cheng X: MiR-582-5p is upregulated in patients with active tuberculosis and inhibits apoptosis of monocytes by targeting FOXO1. PloS One 8: e78381, 2013.

30. Wu J, Lu C, Diao N, et al: Analysis of microRNA expression profiling identifies miR-155 and miR-155* as potential diagnostic markers for active tuberculosis: A preliminary study. Hum Immunol 73: 31-37, 2012. 